\title{
16. Sozioökonomischer Status und freiwilliges Engagement
}

\author{
Julia Simonson \& Nicole Hameister
}

\section{Kernaussagen}

Sowohl das schulische als auch das berufliche Ausbildungsniveau hängen mit freiwilligem Engagement zusammen. Personen, die einen Haupt- oder Realschulabschluss sowie eine abgeschlossene Berufsausbildung haben, engagieren sich zu 36,2 Prozent; die Engagementquote von Personen, die einen Haupt- oder Realschulabschluss haben, jedoch keine Berufsausbildung, beträgt 23,5 Prozent. Die höchsten Anteile Engagierter sind bei Personen mit einem abgeschlossenen Fachhochschul- oder Universitätsstudium (54,1 Prozent) zu finden.

Erwerbstätige sowie Schülerinnen und Schüler engagieren sich zu höheren Anteilen als Rentnerinnen und Rentner, Arbeitslose und sonstige Nicht-Erwerbstätige. Dabei engagieren sich in Teilzeit und geringfügig Beschäftigte (51,1 Prozent) anteilig häufiger als Personen, die in Vollzeit erwerbstätig sind (46,7 Prozent).

Die Engagementquote von Menschen, die in Einpersonenhaushalten leben, ist deutlich niedriger als die von Personen in Mehrpersonenhaushalten. Den höchsten Anteil freiwillig Engagierter weist die Gruppe von Personen auf, die in Mehrpersonenhaushalten mit ihrer Partnerin oder ihrem Partner sowie mit eigenen minderjährigen Kindern leben (54,9 Prozent).

Die selbst eingeschätzte Einkommenssituation wirkt sich auf freiwilliges Engagement aus. Personen, die ihre finanzielle Lage als sehr gut einschätzen, engagieren sich mit 50,0 Prozent zu einem fast doppelt so hohen Anteil wie Personen, die ihre finanzielle Lage als sehr schlecht einschätzen (26,9 Prozent).

Die betrachteten sozioökonomischen Faktoren wirken kumulativ und sie wirken auch bei Einbezug weiterer Merkmale auf freiwilliges Engagement. Die Wahrscheinlichkeit sich freiwillig zu engagieren sinkt, wenn ungünstige Ressourcenausstattungen wie beispielsweise ein niedriger Bildungsstatus und Arbeitslosigkeit zusammentreffen, und sie steigt, wenn förderliche Ressourcen aufeinandertreffen.

\subsection{Einleitung}

Freiwilliges Engagement ist an Voraussetzungen geknüpft: Die bisherige Forschung zum Engagement hat gezeigt, dass ein hoher sozioökonomischer Status in der Regel förderlich für die Ausübung einer freiwilligen Tätigkeit ist (siehe beispielsweise Gensicke 2015; Schmidt
2014). Personen aus höheren Schichten sind deutlich häufiger und auch dauerhafter engagiert als Personen aus niedrigeren sozialen Schichten (Simonson, Hagen, Vogel \& Motel-Klingebiel 2013). Es bestehen also je nach sozioökonomischer Position unterschiedliche

(C) Der/die Autor(en) 2017

J. Simonson et al. (Hrsg.), Freiwilliges Engagement in

Deutschland, Empirische Studien zum bürgerschaftlichen

Engagement, DOI 10.1007/978-3-658-12644-5_17 
Zugangschancen zum freiwilligen Engagement.

Insbesondere die Bildung scheint von Bedeutung zu sein: Zahlreiche Studien zeigen, dass sich höher gebildete Personen zu größeren Anteilen engagieren als Personen mit niedriger Bildung (zum Beispiel Gensicke 2015; Erlinghagen 2008; Naumann \& Romeu Gordo 2010). Der Bildungsstatus kann dabei für ein Bündel unterschiedlicher Einflussfaktoren stehen. Höhergebildete haben häufig bessere materielle und immaterielle Ressourcen, die für die Ausübung eines freiwilligen Engagements notwendig sind, sie verfügen über bessere Informationen, wie und wo sie sich engagieren können und werden vielleicht auch häufiger für eine freiwillige Tätigkeit angeworben. Schließlich können auch bildungs- und schichtspezifisch unterschiedliche Wertvorstellungen und Prägungen einen Einfluss haben.

Bislang wurde insbesondere der Einfluss des schulischen Bildungsniveaus auf das freiwillige Engagement untersucht. Wir gehen davon aus, dass darüber hinaus auch die berufliche Bildung wichtig für die Frage sein kann, ob man sich engagiert oder nicht, da diese einerseits einen relevanten Einfluss auf die aktuelle Lebenssituation und die ökonomische Ressourcenlage hat und andererseits bedeutsam für die Frage der Passung von freiwilligen Tätigkeiten sein kann. So sind Personen mit einem akademischen Abschluss möglicherweise nicht nur auf dem Arbeitsmarkt, sondern auch im freiwilligen Engagement, ,gefragter' als Personen, die keine entsprechende Ausbildung vorweisen können.

Auch der Erwerbsstatus kann von Bedeutung für das freiwillige Engagement sein. Über die Erwerbsarbeit können sich zusätzliche Gelegenheiten für freiwilliges Engagement ergeben, beispielsweise für Tätigkeiten in Beiräten oder beruflichen Interessenvertretungen - oder einfach, da man häufiger mit anderen Menschen in Kontakt kommt, die einen für eine freiwillige Tätigkeit gewinnen. Tatsächlich sind die Anteile Engagierter unter erwerbstätigen Personen höher als unter Nicht-Erwerbstätigen. Insbesondere Personen, die arbeitslos sind, engagieren sich zu geringeren Anteilen als andere (Strauß 2009). Dies könnte zum einen daran liegen, dass mit dem Verlust der Erwerbstätigkeit auch wichtige Gelegenheitsstrukturen für das Engagement verloren gehen, zum anderen könnten ähnliche Mechanismen, die ausschlaggebend sind, um eine Arbeitsstelle zu finden, auch für das ,Besetzen' ehrenamtlicher Positionen von Bedeutung sein. Darüber hinaus verfügen Arbeitslose häufig nicht über ausreichende finanzielle Ressourcen, um die Kosten zu tragen, die mit der Übernahme einer freiwilligen Tätigkeit verbunden sein können (siehe Kapitel 14).

Gelegenheiten für freiwilliges Engagement können auch mit der Familienstruktur und der Haushaltskonstellation variieren. Das Vorhandensein von Kindern kann Anknüpfungspunkte für freiwilliges Engagement bieten (zum Beispiel im Bereich Schule und Kindergarten, aber auch in Sportvereinen). Gleichzeitig können Kinder - insbesondere im Zusammenspiel mit einer Erwerbsbeteiligung - das Zeitkontingent für freiwilliges Engagement aber auch begrenzen. Darüber hinaus können sich Möglichkeiten für freiwilliges Engagement über die Partnerin beziehungsweise den Partner sowie über andere Haushaltsmitglieder ergeben.

Schließlich kann die finanzielle Situation ausschlaggebend sein. So können es sich Menschen mit höherem Einkommen eher leisten, sich freiwillig zu engagieren, wenn dabei Kosten entstehen, zum Beispiel über Mitgliedsbeiträge oder Fahrtkosten. Insbesondere armutsgefährdete Personen (Böhnke 2008) engagieren sich seltener als andere. Möglicherweise spielen dabei nicht nur die mangelnden finanziellen Mittel für Fahrtkosten, Mitgliedsbeiträge und andere Auslagen eine Rolle, sondern auch die Konkurrenz von freiwilligen beziehungsweise ehrenamtlichen und beruflichen Tätigkeiten. In finanziellen Notsituationen wird vermutlich zunächst der Gewinnorientierung Vorrang gegeben, um den Lebensstandard zu sichern. Das Ausüben einer freiwilligen und unbezahlten Tätigkeit tritt demgegenüber in den Hintergrund. 
Die beschriebenen sozioökonomischen Faktoren können nicht isoliert voneinander betrachtet werden, sondern sind miteinander verzahnt. So beeinflusst beispielsweise das Bildungsniveau den Erwerbsstatus und die finanzielle Situation. Gleichzeitig ist das Einkommen im Zusammenhang mit der Haushaltskonstellation zu sehen. Inwieweit ein Einkommen als ausreichend bewertet wird, hängt entscheidend davon $\mathrm{ab}$, für wie viele Personen man sorgen muss, ob also beispielsweise auch Partnerin beziehungsweise Partner und Kinder daran teilhaben. Gleichzeitig können sich durch das Zusammenleben in einem Haushalt aber auch Einspareffekte ergeben, da bestimmte Güter des alltäglichen Bedarfs nur einmal pro Haushalt und nicht einmal pro Person angeschafft werden müssen.

Sozioökonomische Aspekte können dazu beitragen, unterschiedliche Engagementquoten zwischen den Geschlechtern und zwischen Altersgruppen zu erklären. Die allgemeine Engagementquote ist bei Frauen niedriger als bei Männern (siehe Kapitel 3). Dies kann zum Teil auf den sich unterscheidenden sozioökonomischen Status von Frauen und Männern zurückgeführt werden, der unterschiedlich gute Ressourcen für freiwilliges Engagement, beispielsweise hinsichtlich der materiellen Situation beinhaltet. Zum Teil können geschlechtsspezifische Unterschiede im Engagement aber auch durch die geschlechtsspezifische Arbeitsteilung erklärt werden. So stellt sich die Frage nach der Vereinbarkeit mit familialen und beruflichen Aufgaben und Verpflichtungen für die Geschlechter häufig in unterschiedlicher Weise (vgl. Alscher 2010). Frauen sind neben ihrer Berufstätigkeit in den meisten heterosexuellen Paarbeziehungen und Familien mit Kindern stärker für die reproduktiven Hausund Pflegearbeiten zuständig. Zwar entstehen in diesen Bereichen durchaus Möglichkeiten für freiwilliges Engagement (beispielsweise in Schule oder Kindergarten), allerdings haben Frauen neben ihren Verantwortlichkeiten für Beruf und Familie möglicherweise weniger zeitliche Ressourcen für eine freiwillige Tätigkeit als Männer. Freiwilliges Engagement ist daher auch in Bezug zum familiären Bezugsrahmen und zur Haushaltskonstellation sowie zum Erwerbsleben zu setzen (Zierau 2001). Auch die geringere Beteiligung Älterer kann zumindest teilweise auf die im Ruhestand geringer werdenden Gelegenheitsstrukturen für freiwilliges Engagement zurückgeführt werden, darüber hinaus sind hier aber auch weitere Faktoren wie beispielsweise der Gesundheitszustand von Bedeutung (siehe Kapitel 17).

Schließlich können sich die Effekte sozioökonomischer Faktoren zwischen Frauen und Männern sowie zwischen Angehörigen unterschiedlicher Altersgruppen unterscheiden. So kann beispielsweise eine Phase der Nicht-Erwerbstätigkeit von Frauen und Männern aufgrund geschlechtsspezifisch geprägter Rollenmuster und Erwartungen unterschiedlich wahrgenommen und bewertet werden und insofern auch andere Auswirkungen für die Ausübung freiwilliger Tätigkeiten nach sich ziehen.

In diesem Kapitel wird untersucht, welche Bedeutung den sozioökonomischen Faktoren Schul- und Berufsbildung, Erwerbsstatus, Haushaltskonstellation und finanzielle Situation hinsichtlich der Ausübung eines freiwilligen Engagements zukommt. Dabei wird betrachtet, welchen Einfluss die jeweiligen Merkmale auf die Wahrscheinlichkeit haben, sich freiwillig $\mathrm{zu}$ engagieren, und inwieweit sie gruppenspezifisch wirken, also beispielsweise ob sich eine schlechte Einkommenssituation bei Frauen in anderer Weise auf das Engagement auswirkt als bei Männern. Im Einzelnen werden folgende Forschungsfragen untersucht:

1. Unterscheiden sich Menschen mit unterschiedlichen sozioökonomischen Ressourcen (schulische und berufliche Bildung, Erwerbsstatus, Haushaltskonstellation und Einkommenssituation) in ihrem Engagement?

2. Weisen schulische und berufliche Bildung, Erwerbsstatus, Haushaltskonstellation und Einkommenssituation bei Frauen und Männern sowie bei Angehörigen unterschiedli- 
cher Altersgruppen unterschiedliche Zusammenhänge mit Engagement auf?

3. Ist die Bedeutung von schulischer und beruflicher Bildung, Erwerbsstatus, Haushalts- konstellation und Einkommenssituation auch dann nachweisbar, wenn andere Aspekte der Lebenssituation von Menschen berücksichtigt werden (multivariate Betrachtung)?

\subsection{Datengrundlage}

Die in diesem Kapitel berichteten deskriptiven Befunde basieren auf gewichteten Analysen. Für die Gewichtung wurden in allen Erhebungsjahren dieselben Gewichtungsmerkmale berücksichtigt. Dabei handelt es sich um die Designgewichtung, die sich auf die Auswahlwahrscheinlichkeit einer Person bezieht (Haushaltsgröße, Zahl der Festnetznummern und, nur für das Jahr 2014, auch die Zahl der Mobilfunknummern) und Gewichtungsmerkmale, die Abweichungen der Stichprobenverteilung von der Grundgesamtheit in bestimmten Dimensionen ausgleichen (Bundesland, Gemeindegrößenklassen, Geschlecht, Altersgruppen). Von einer Hinzunahme weiterer Gewichtungsmerkmale wie etwa des Bildungsstandes haben wir abgesehen, um die Vergleichbarkeit mit den bisher veröffentlichten Berichten zu erhalten.

Bildungsstatus nach ISCED (International Standard Classification for Education) (Organisation for Economic Co-operation and Development (OECD) 1999): Im Freiwilligensurvey 2014 wurden die Befragten sehr differenziert nach ihren bisher abgeschlossenen Schul- und Berufsausbildungen gefragt. Damit ist es im Freiwilligensurvey 2014 erstmals möglich, nicht nur das schulische, sondern auch das berufliche Bildungsniveau im Zusammenhang mit freiwilligem Engagement zu betrachten. Alle Befragten wurden gebeten, eine Reihe von Fragen zu ihren bisher erreichten Schul- und Ausbildungsabschlüssen zu beantworten. Die Kernfrage zur schulischen Bildung lautete:

Welchen höchsten Schulabschluss haben Sie? Volksoder Hauptschulabschluss, Abschluss Polytechni- sche Oberschule (POS) 8. Klasse; Mittlere Reife, Abschluss Polytechnische Oberschule (POS) 10. Klasse; Fachhochschulreife (Abschluss in der Regel nach 12. bzw. 11. Klasse (wenn kein Abitur)), Abschluss an Berufsschulen, Fachkollegs, Fachabitur; Abitur, Abschluss einer erweiterten Oberschule (EOS), Hochschulreife; einen anderen Schulabschluss; keinen Schulabschluss?

Bei Personen, die die Schule zuletzt nicht in Deutschland besucht haben, wurden zwei Alternativfragen gestellt. Zum einen wurde gefragt:

Wie viele Jahre haben Sie die Schule besucht? (Offene Antwortmöglichkeit in Jahren)

Zum anderen wurde nach dem erreichten $\mathrm{Ab}$ schluss gefragt:

Mit welchem Abschluss haben Sie die Schule beendet? Haben Sie die Schule ohne Abschluss verlassen; die Pflichtschule mit Abschluss beendet; eine weiterführende Schule mit Abschluss beendet?

Hinsichtlich der beruflichen Ausbildung wurden ebenfalls mehrere Fragen gestellt. Bei Personen, die angegeben haben, eine berufliche Ausbildung oder ein Studium in Deutschland absolviert zu haben, wurde gefragt:

Welchen höchsten Ausbildungsabschluss haben Sie: Abschluss einer Lehre, das heißt eine betriebliche oder schulische Berufsausbildung; Abschluss an einer Fachschule (Meisterschule, Technikerschule, Berufsakademie, Verwaltungshochschule); Beamtenausbildung für den einfachen oder mittleren 
Dienst; Beamtenausbildung für den gehobenen Dienst; Hochschul- oder Universitätsabschluss wie Bachelor, Master, Diplom, Magister, Staatsexamen oder Beamtenausbildung für den höheren Dienst; Promotion oder Habilitation; einen anderen $A b$ schluss?

Personen, die eine Ausbildung oder ein Studium in einem anderen Land abgeschlossen haben, wurden gefragt:

Welchen höchsten Ausbildungsabschluss haben Sie: Abschluss einer Berufsausbildung; Fachhochschul- oder Universitätsabschluss; sonstiges?

Durch Kombination der Antworten ist es möglich, für nahezu jede Teilnehmerin und jeden
Teilnehmer im Freiwilligensurvey 2014 den Bildungsstand gemäß der international gebräuchlichen Klassifizierung ISCED 1997 (OECD 1999) zu generieren. Die hier vorgenommene Umsetzung orientiert sich am Vorgehen im $\mathrm{Mi}$ krozensus sowie in weiteren etablierten Studien wie dem Nationalen Bildungspanel (NEPS) und dem Sozio-oekonomischen Panel (SOEP). Aus Gründen der Übersichtlichkeit werden die neun üblicherweise verwendeten ISCED-Kategorien für die Analysen in diesem Kapitel zu sieben zusammengefasst. Diese sieben Kategorien beinhalten die in Tabelle 16-1 dargestellten Kombinationen von derzeitig höchstem Schul- und Berufsabschluss.

Tabelle 16-1: Bildungsstatus nach ISCED: Kombination von Schul- und Berufsabschluss

\begin{tabular}{|c|c|c|}
\hline $\begin{array}{l}\text { ISCED-Stufen im FWS } 2014 \\
\text { (Original-ISCED-Level) }\end{array}$ & Schulabschluss & Beruflicher Abschluss \\
\hline $\begin{array}{l}\text { Noch Schülerin/Schüler } \\
\text { (0/1 - Vorschulische Erziehung/ } \\
\text { Grundbildung) }\end{array}$ & $\begin{array}{l}\text { Derzeit noch Besuch einer } \\
\text { allgemeinbildenden Schule }\end{array}$ & \\
\hline $\begin{array}{l}\text { Haupt-/Realschulabschluss } \\
\text { ohne berufliche Ausbildung } \\
\text { ( } 2 \text { - Sekundarbildung I) }\end{array}$ & $\begin{array}{l}\text { Abschluss an einer Haupt- } \\
\text { oder Realschule bzw. ähnliche } \\
\text { Ausbildung }\end{array}$ & $\begin{array}{l}\text { Kein Abschluss einer } \\
\text { beruflichen Ausbildung }\end{array}$ \\
\hline $\begin{array}{l}\text { FH-Reife/Abitur ohne berufliche } \\
\text { Ausbildung } \\
\text { (3a-Sekundarbildung II) }\end{array}$ & $\begin{array}{l}\text { Abschluss einer Fachhochschul- } \\
\text { reife oder des Abiturs bzw. } \\
\text { ähnliche Ausbildung }\end{array}$ & $\begin{array}{l}\text { Kein Abschluss einer } \\
\text { beruflichen Ausbildung }\end{array}$ \\
\hline $\begin{array}{l}\text { Haupt-/Realschulabschluss und } \\
\text { berufliche Ausbildung } \\
\text { (3b/3c - Duale Berufsausbildung) }\end{array}$ & $\begin{array}{l}\text { Abschluss an einer Haupt- } \\
\text { oder Realschule bzw. ähnliche } \\
\text { Ausbildung }\end{array}$ & $\begin{array}{l}\text { Abschluss einer Lehre oder } \\
\text { ähnliche Ausbildung }\end{array}$ \\
\hline $\begin{array}{l}\text { FH-Reife/Abitur und berufliche } \\
\text { Ausbildung } \\
\text { (4-Postsekundäre Bildung) }\end{array}$ & $\begin{array}{l}\text { Abschluss einer Fachhochschul- } \\
\text { reife oder des Abiturs bzw. } \\
\text { ähnliche Ausbildung }\end{array}$ & $\begin{array}{l}\text { Abschluss einer Lehre oder } \\
\text { ähnliche Ausbildung }\end{array}$ \\
\hline $\begin{array}{l}\text { Abschluss Berufs-/Fachakademie } \\
\text { ( } 5 \mathrm{~b}-\text { Tertiäre Bildung mit Praxis- } \\
\text { bezug) }\end{array}$ & & $\begin{array}{l}\text { Abschluss an Fach- oder Berufs- } \\
\text { akademie, Meisterabschluss, } \\
\text { Verwaltungshochschule oder } \\
\text { ähnliche Ausbildung }\end{array}$ \\
\hline $\begin{array}{l}\text { FH-/Universitätsabschluss } \\
\text { (5a/6 - Tertiäre Bildung/Forschungs- } \\
\text { qualifikation) }\end{array}$ & & $\begin{array}{l}\text { Abschluss eines Studiums an } \\
\text { Fachhochschule oder Universität, } \\
\text { auch Promotion oder Habilitation }\end{array}$ \\
\hline
\end{tabular}

Quelle: FWS 2014, eigene Darstellung (DZA). 
Tabelle 16-2 zeigt die Verteilung der ISCED-Bildungsstufen im Freiwilligensurvey 2014 insgesamt und nach Geschlecht differenziert. Etwa ein Drittel der Teilnehmerinnen und Teilnehmer des Freiwilligensurveys verfügt über einen Hauptoder Realschulabschluss sowie eine berufliche Ausbildung. Ein knappes Viertel hat ein Studium an einer Fachhochschule oder Universität abgeschlossen. Jede achte Person hat einen Abschluss einer Fach- oder Berufsakademie, einer Verwaltungshochschule oder einen äquivalenten Abschluss. Etwas seltener sind der Abschluss von
Haupt- oder Realschule beziehungsweise deren Äquivalent ohne Berufsausbildung, von Abitur beziehungsweise Fachhochschulreife ohne Berufsausbildung sowie die Kombination von Abitur beziehungsweise Fachhochschulreife und einer beruflichen Ausbildung. Jede zwanzigste Person geht noch zur Schule. Unterschiede zwischen den Geschlechtern zeigen sich insbesondere beim Fachhochschul-/Universitätsabschluss (bei Männern verbreiteter) und beim Abschluss von Haupt- oder Realschule ohne Berufsabschluss (bei Frauen verbreiteter).

Tabelle 16-2: Verteilung der ISCED-Bildungsstufen 2014

\begin{tabular}{lccc}
\hline & Frauen & Männer & Gesamt \\
\hline Noch Schülerin/Schüler & 4,7 & 5,0 & 4,8 \\
\hline Haupt-/Realschulabschluss ohne berufliche Ausbildung & 11,7 & 7,4 & 9,6 \\
\hline FH-Reife/Abitur ohne berufliche Ausbildung & 8,0 & 7,7 & 7,9 \\
\hline Haupt-/Realschulabschluss und berufliche Ausbildung & 34,8 & 32,0 & 33,4 \\
\hline FH-Reife/Abitur und berufliche Ausbildung & 8,8 & 7,2 & 8,0 \\
\hline Abschluss Berufs-/Fachakademie & 11,1 & 13,8 & 12,4 \\
\hline FH-/Universitätsabschluss & 21,0 & 26,9 & 23,9 \\
\hline
\end{tabular}

Quelle: FWS 2014, gewichtet, eigene Berechnungen (DZA). Basis: Alle Befragten mit gültigen Angaben zu schulischer und beruflicher Bildung $(n=28.682)$.

Dargestellt sind Spaltenprozente (die Prozentzahlen innerhalb einer Spalte summieren sich zu 100 Prozent).

Erwerbsstatus: Im Freiwilligensurvey 2014 werden von allen Befragten in mehreren Stufen umfangreiche Informationen zur beruflichen Tätigkeit und zur derzeitigen Haupttätigkeit erhoben, die eine differenzierte Zuschreibung des aktuellen Erwerbsstatus erlauben. Die zentrale Frage hierzu ist:

Sind Sie derzeit: erwerbstätig; arbeitslos gemeldet; Schüler/in, in Ausbildung, im Studium; im Mutterschutz/in Elternzeit; Hausfrau, Hausmann; Rentner/in, Pensionär/in; im freiwilligen Wehrdienst; in einem Freiwilligendienst; in Umschulung oder Weiterbildung; aus sonstigen Gründen nicht erwerbstätig?
Erwerbstätigen Personen wurden außerdem zwei Fragen zum Umfang der Erwerbstätigkeit gestellt:

Handelt es sich um: eine Vollzeitbeschäftigung; eine Teilzeitbeschäftigung; eine geringfügige Beschäftigung, zum Beispiel einen Minijob?

Wie viele Stunden beträgt im Durchschnitt Ihre wöchentliche Arbeitszeit? Bitte zählen Sie Überstunden mit dazu.

Für die Analysen in diesem Kapitel sind die Informationen aus diesen Fragen zu sechs Kategorien zusammengefasst worden: 
1. Erwerbstätig in Vollzeit (35 Wochenstunden und mehr)

2. Erwerbstätig in Teilzeit oder in geringfügigem Umfang

3. Nicht erwerbstätig und arbeitslos gemeldet

4. Nicht erwerbstätig, weil Rentner/-in oder in Pension

5. Nicht erwerbstätig, weil in Ausbildung (Schule, Berufsausbildung, Studium)

6. Nicht erwerbstätig, aus sonstigen Gründen (inkl. Freiwilligendienst, freiwilliger Wehrdienst, Weiterbildung, Mutterschutz/ Elternzeit, Hausmann/-frau etc.)
Tabelle 16-3 zeigt die Verteilung dieser sechs Erwerbsstatuskategorien im Freiwilligensurvey 2014 insgesamt und nach Geschlecht differenziert. Von allen Befragten sind mehr als ein Drittel in Vollzeit erwerbstätig, allerdings Frauen (23,4 Prozent) zu deutlich geringeren Anteilen als Männer (51,6 Prozent). Dies kehrt sich bei der Teilzeiterwerbstätigkeit (inkl. geringfügige Erwerbstätigkeit) um: 20,5 Prozent der Frauen, aber nur 3,6 Prozent der Männer arbeiten in Teilzeit oder sind geringfügig beschäftigt. Insgesamt ein gutes Viertel der Personen befindet sich im Ruhestand (Frauen zu etwas größeren Anteilen als Männer).

Tabelle 16-3: Verteilung der Erwerbsstatuskategorien 2014

\begin{tabular}{lccc}
\hline & Frauen & Männer & Gesamt \\
\hline Erwerbstätig in Vollzeit & 23,4 & 51,6 & 37,1 \\
\hline Erwerbstätig in Teilzeit/geringfügig erwerbstätig & 20,5 & 3,6 & 12,3 \\
\hline Nicht erwerbstätig: arbeitslos & 3,1 & 4,0 & 3,6 \\
\hline Nicht erwerbstätig: in Rente oder Pension & 28,1 & 24,3 & 26,3 \\
\hline Nicht erwerbstätig: in Ausbildung & 12,0 & 12,9 & 12,5 \\
\hline Nicht erwerbstätig: sonstige Gründe & 12,8 & 3,6 & 8,3 \\
\hline
\end{tabular}

Quelle: FWS 2014, gewichtet, eigene Berechnungen (DZA). Basis: Alle Befragten mit gültigen Angaben zum Erwerbsstatus $(n=28.591)$.

Dargestellt sind Spaltenprozente (die Prozentzahlen innerhalb einer Spalte summieren sich zu 100 Prozent). 
Haushaltskonstellation: Alle im Freiwilligensurvey 2014 befragten Personen geben Auskunft über die Zusammensetzung des Haushaltes, in dem sie leben. Dazu gehört vor allem die Anzahl der Haushaltsmitglieder, die mit folgender Frageformulierung erfasst wird:

Wie viele Personen leben insgesamt in ihrem Haushalt? Bitte denken Sie daran, sich selbst mitzuzählen.
Im Anschluss wird unter anderem nachgefragt, ob Personen unter 18 Jahren und der/die Ehebeziehungsweise Lebenspartner/-in im Haushalt leben. Aus diesen Informationen wird die Variable zur Haushaltskonstellation gebildet, die in diesem Kapitel in folgenden fünf Kategorien verwendet wird (Tabelle 16-4).

Tabelle 16-4: Haushaltskonstellation 2014

\begin{tabular}{|c|c|c|c|c|}
\hline $\begin{array}{l}\text { Haushaltskonstellation im } \\
\text { FWS } 2014\end{array}$ & $\begin{array}{l}\text { Anzahl } \\
\text { der Personen } \\
\text { im Haushalt }\end{array}$ & $\begin{array}{l}\text { Ehe- bzw. } \\
\text { Lebens- } \\
\text { partner/-in } \\
\text { im Haus- } \\
\text { halt? }\end{array}$ & $\begin{array}{l}\text { (Weitere) } \\
\text { Personen } \\
\text { unter } 18 \\
\text { Jahren im } \\
\text { Haushalt? }\end{array}$ & Konkretes Beispiel \\
\hline Einpersonenhaushalt & eine & nein & nein & Alleinlebende/r \\
\hline $\begin{array}{l}\text { Alleinlebend in } \\
\text { Mehrpersonenhaushalt }\end{array}$ & zwei und mehr & nein & nein & $\begin{array}{l}\text { Jugendliche/r im Haushalt der Eltern. } \\
\text { Mitglied einer Wohngemeinschaft. } \\
\text { Alleinstehendes Elternteil im Haushalt } \\
\text { der erwachsenen Kinder }\end{array}$ \\
\hline Ohne Partner/in, mit Kind & zwei und mehr & nein & ja & $\begin{array}{l}\text { Alleinerziehende/r mit minderjähri- } \\
\text { gem Kind/Kindern }\end{array}$ \\
\hline Mit Partner/in, ohne Kind & zwei und mehr & ja & nein & $\begin{array}{l}\text { Ehepaar oder Partnerschaft ohne } \\
\text { minderjähriges Kind/Kinder } \\
\text { im Haushalt }\end{array}$ \\
\hline Mit Partner/in, mit Kind & drei und mehr & ja & ja & $\begin{array}{l}\text { Ehepaar oder Partnerschaft mit } \\
\text { minderjährigem Kind/Kindern } \\
\text { im Haushalt }\end{array}$ \\
\hline
\end{tabular}

Quelle: FWS 2014. Eigene Darstellung (DZA). 
Tabelle 16-5 zeigt die Verteilung dieser fünf Kategorien der Haushaltskonstellation im Freiwilligensurvey 2014 insgesamt und nach Geschlecht differenziert. Knapp zwei Fünftel der Personen leben zusammen mit der Partnerin beziehungsweise dem Partner, aber ohne ein minderjähriges Kind im Haushalt. Jeweils ein Fünftel lebt mit der Partnerin beziehungsweise dem Partner sowie mindestens einem minderjährigen Kind im Haushalt oder aber alleine in einem Einpersonenhaushalt. 13,2 Prozent leben ohne Partnerin oder Partner in einem Mehrpersonenhaushalt (Männer zu etwas höheren Anteilen als Frauen) und nur 3,0 Prozent ohne Partner oder Partnerin, aber gemeinsam mit mindestens einem Kind (Frauen anteilig häufiger als Männer). Von 1.376 Personen liegt entweder zum Partnerstatus oder zu Kindern im Haushalt keine gültige Information vor, diese Fälle werden bei der Betrachtung der Haushaltskonstellation in diesem Kapitel als fehlend von der Analyse ausgeschlossen.

Tabelle 16-5: Verteilung der Haushaltskonstellationskategorien 2014

\begin{tabular}{lccc}
\hline & Frauen & Männer & Gesamt \\
\hline Einpersonenhaushalt & 24,9 & 21,0 & 23,0 \\
\hline Alleinlebend in Mehrpersonenhaushalt & 12,0 & 14,6 & 13,2 \\
\hline Ohne Partner/in, mit Kind & 4,7 & 1,3 & 3,0 \\
\hline Mit Partner/in, ohne Kind & 39,0 & 42,7 & 40,8 \\
\hline Mit Partner/in, mit Kind & 19,5 & 20,5 & 20,0 \\
\hline
\end{tabular}

Quelle: FWS 2014, gewichtet, eigene Berechnungen (DZA). Basis: Alle Befragten mit gültigen Angaben zur Haushaltskonstellation $(n=27.277)$.

Dargestellt sind Spaltenprozente (die Prozentzahlen innerhalb einer Spalte summieren sich zu 100 Prozent).

Subjektive Einkommenssituation: Am Schluss des Interviews werden die Befragten im Freiwilligensurvey 2014 noch um eine Einschätzung ihrer finanziellen Situation gebeten:
Wie würden Sie heute Ihre finanzielle Situation einstufen? Als sehr gut; eher gut; mittel; eher schlecht; sehr schlecht? 
Für die Darstellung der Engagementquoten in Abhängigkeit von der subjektiven Einkommenssituation in diesem Kapitel bleiben wir bei dieser fünffachen Abstufung. Im multivariaten Modell am Ende des Kapitels fassen wir die Kategorien ,sehr gut und ,gut ${ }^{\text {zu }}$, gute Einkommenssituation` zusammen und kontrastieren diese mit den anderen drei Kategorien, die zu ,mittel bis schlechte Einkommenssituation' zusammengefasst sind. Tabelle 16-6 zeigt die Verteilung der fünf Kategorien der subjektiven Einkommenssituation im Freiwilligensurvey 2014 insgesamt und nach Geschlecht differenziert.

Tabelle 16-6: Verteilung der subjektiven Einkommenssituation 2014

\begin{tabular}{lccc}
\hline & Frauen & Männer & Gesamt \\
\hline Sehr gut & 13,1 & 14,5 & 13,8 \\
\hline Gut & 35,7 & 37,1 & 36,4 \\
Mittel & 41,1 & 37,6 & 39,4 \\
\hline Eher schlecht & 7,2 & 7,8 & 7,5 \\
\hline Sehr schlecht & 3,0 & 3,1 & 3,0 \\
\hline
\end{tabular}

Quelle: FWS 2014, gewichtet, eigene Berechnungen (DZA). Basis: Alle Befragten mit gültigen Angaben zur finanziellen Situation ( $n=28.414)$.

Dargestellt sind Spaltenprozente (die Prozentzahlen innerhalb einer Spalte summieren sich zu 100 Prozent).

Knapp zwei Fünftel der Personen bewerten die eigene finanzielle Situation als mittel und über ein Drittel bewertet sie als gut. 13,8 Prozent bewerten die eigene finanzielle Situation sogar als sehr gut. Deutlich seltener ist die Einschätzung einer eher schlechten (7,5 Prozent) beziehungsweise sehr schlechten finanziellen Situation (3,0
Prozent). Frauen und Männer unterscheiden sich hinsichtlich der selbsteingeschätzten finanziellen Situation nur wenig, allerdings geben Frauen anteilig etwas seltener an, die Situation sei gut oder sehr gut und häufiger ihre finanzielle Situation sei mittel. 


\subsection{Schul- und Berufsausbildung und freiwilliges Engagement}

In Kapitel 3 dieses Berichts wird gezeigt, dass die Ausübung freiwilligen Engagements deutlich zwischen Personen mit unterschiedlicher Schulbildung variiert, wobei sich Personen mit höherer Bildung auch zu größeren Anteilen engagieren. Im Folgenden soll der Fokus ausgeweitet und auch die berufliche Bildung mit einbezogen werden. In Abbildung 16-1 sind die Quoten freiwilligen Engagements nach Schulund Berufsbildungsgruppen dargestellt. Neben den Personen, die noch zur Schule gehen $(54,8$ Prozent), engagieren sich Personen mit einem abgeschlossenen Fachhochschul- oder Universitätsstudium zu den höchsten Anteilen (54,1 Prozent). Überdurchschnittliche Engagementraten weisen darüber hinaus Personen mit Fachhochschulreife oder Abitur, aber ohne Berufsausbildung (49,4 Prozent) sowie Personen mit dem Abschluss einer Berufs- oder Fachakademie auf (49,9 Prozent). Äußerst geringe Anteile freiwillig Engagierter finden wir bei Personen mit einer niedrigen oder mittleren Schulbildung (Hauptoder Realschulabschluss) ohne berufliche Ausbildung (23,5 Prozent).

\section{Abbildung 16-1: Anteile freiwillig Engagierter 2014, nach schulischer und beruflicher Bildung}

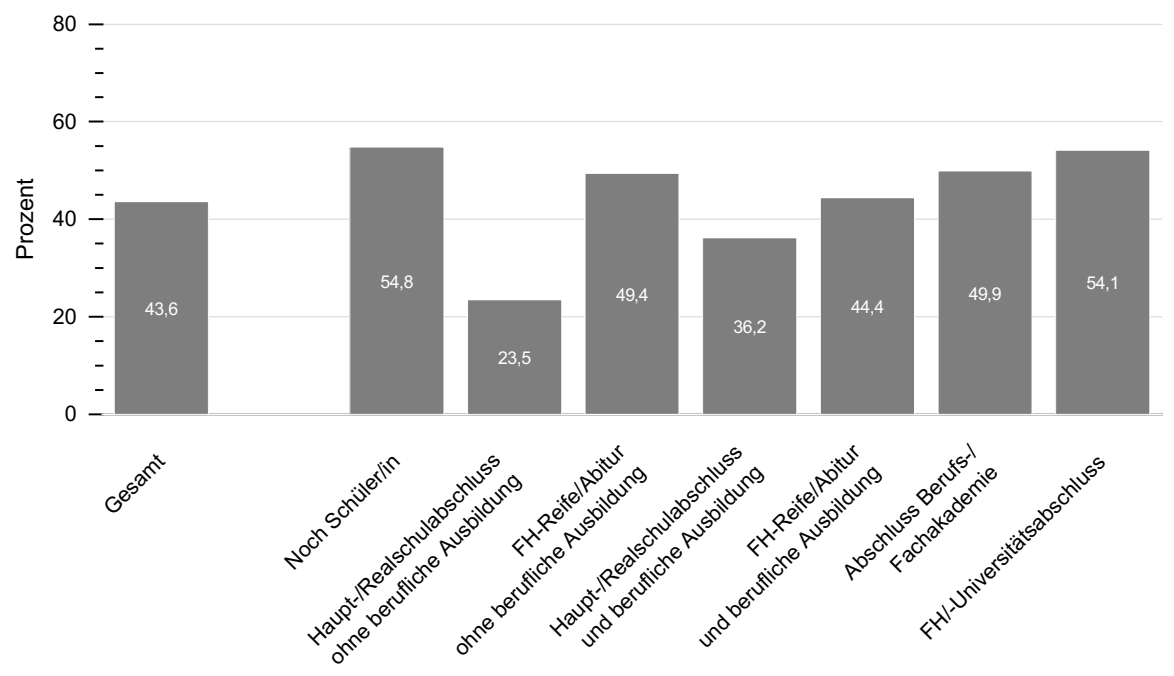

Quelle: FWS 2014, gewichtet. eigene Berechnungen (DZA). Basis: Alle Befragten ( $n=28.681$ ).

Frauen und Männer engagieren sich innerhalb der Bildungsgruppen überwiegend $\mathrm{zu}$ sehr ähnlichen Anteilen. Signifikante Geschlechterunterschiede finden wir nur bei Personen mit Haupt- beziehungsweise Realschulabschluss ohne berufliche Ausbildung sowie bei Personen mit Haupt- beziehungsweise Realschulabschluss mit beruflicher Ausbildung. In beiden Gruppen engagieren sich Männer zu größeren Anteilen als Frauen.

In den Abbildungen 16-1 und 16-2 sehen wir, dass sich Personen, die über Fachhochschulreife/ Abitur und eine Berufsausbildung verfügen, $\mathrm{zu}$ geringeren Anteilen freiwillig engagieren als Personen, die die Fachhochschulreife beziehungsweise das Abitur haben, aber keine Berufsausbil- 
dung. Dies widerspricht auf den ersten Blick dem sonstigen Ergebnis, dass sich Personen mit höherer Bildung häufiger engagieren als Personen mit niedrigerer Bildung. Betrachtet man die Ergebnisse differenziert nach Altersgruppen (siehe Tabelle 16-1 im Online-Anhang), so relativiert sich der Befund: Tatsächlich sind es im direkten Vergleich beider Bildungsgruppen nur die Personen der jüngsten Altersgruppe, die sich zu (deutlich) größeren Anteilen engagieren, wenn sie zwar die (Fach-)Hochschulreife (53,5 Prozent), aber (noch) nicht zusätzlich eine berufliche Ausbildung (40,7 Prozent) abgeschlossen haben. Hierbei dürfte es sich häufig um Studierende handeln. In den anderen Altersgruppen engagieren sich im Vergleich durchgängig diejenigen Personen häufiger, die zusätzlich zur (Fach-)Hochschulreife eine berufliche Ausbildung abgeschlossen haben.

\section{Abbildung 16-2: Anteile freiwillig Engagierter 2014, nach schulischer und beruflicher Bildung und Geschlecht}

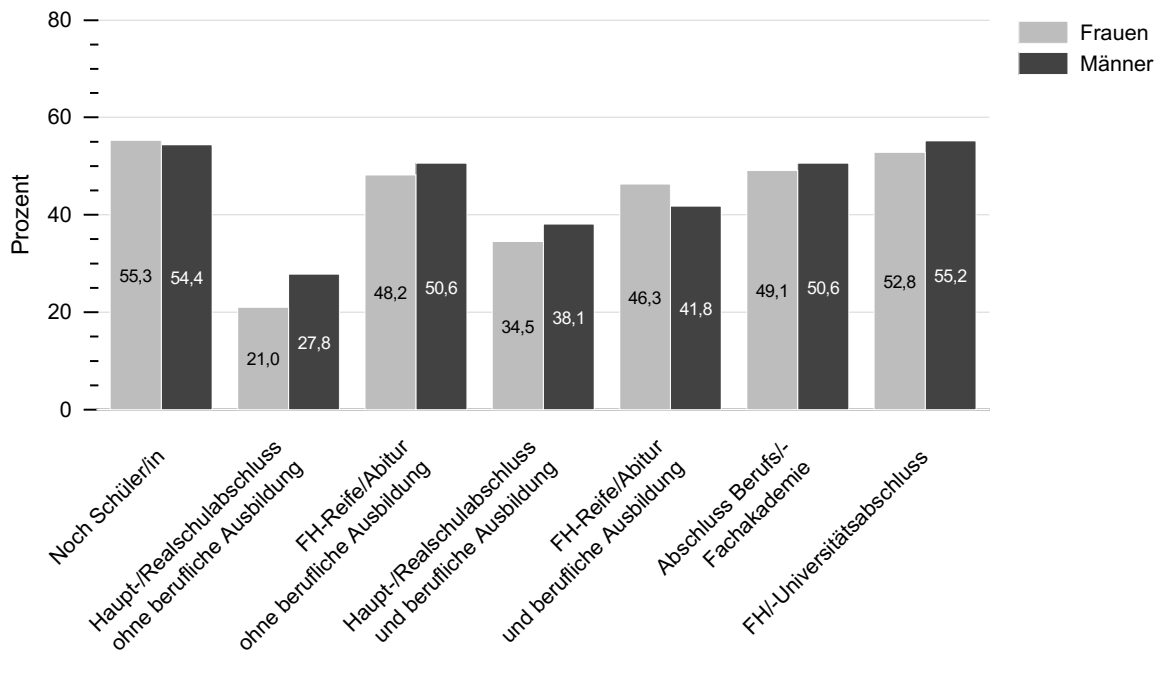

Quelle: FWS 2014, gewichtet, eigene Berechnungen (DZA). Basis: Alle Befragten $(n=28.681$ ). 


\subsection{Erwerbsstatus und freiwilliges Engagement}

Erwerbstätige sowie Personen in Ausbildung engagieren sich zu deutlich höheren Anteilen als Nicht-Erwerbstätige, wobei arbeitslos gemeldete Personen mit 26,1 Prozent die mit Abstand niedrigsten Engagementquoten aufweisen (Abbildung 16-3). Personen im Ruhestand (35,3 Prozent) sowie sonstige Nichterwerbstätige $(38,5$ Prozent) sind demgegenüber etwas häufiger, aber immer noch zu unterdurchschnittlichen Anteilen engagiert. Teilzeit- sowie geringfügig Beschäftigte engagieren sich zu größeren Anteilen als Vollzeitbeschäftigte, was vermutlich auf die größeren Zeitressourcen der Teilzeiterwerbstätigen - bei vergleichbaren Gelegenheitsstrukturen - zurückzuführen ist.

Betrachtet man die Engagementquoten differenziert nach Erwerbsstatus und Geschlecht (Abbildung 16-4), so zeigt sich jedoch, dass dies ausschließlich auf Frauen zutrifft: Während 42,7 Prozent der in Vollzeit erwerbstätigen Frauen einem freiwilligen Engagement nachgehen, sind es bei den in Teilzeit beziehungsweise geringfügig beschäftigten Frauen mit 52,5 Prozent knapp zehn Prozentpunkte mehr. Bei den Männern sind dagegen die Vollzeiterwerbstätigen $\mathrm{zu}$ höheren Anteilen engagiert: 48,7 Prozent gegenüber 42,7 Prozent bei den teilzeit- beziehungsweise geringfügig beschäftigten Männern. Möglicherweise sind bei den Männern die mit einer Vollzeiterwerbstätigkeit einhergehenden Opportunitäten und finanziellen Ressourcen ausschlaggebender für die Ausübung einer freiwilligen Tätigkeit als die mit einer Teilzeitbeschäftigung verbundenen Zeitersparnisse. Dies erscheint vor dem Hintergrund plausibel, dass Frauen häufig mehr Zeit in Haushalt und Kindererziehung investieren, die Zeitressourcen für freiwilliges Engagement bei Vollzeit arbeitenden Frauen also im Durchschnitt möglicherweise knapper sind als bei Männern, die einer Erwerbstätigkeit mit der gleichen Stundenzahl nachgehen. Geschlechterunterschiede zeigen sich darüber hinaus bei Arbeitslosen sowie bei Personen im Ruhestand, wobei jeweils Frauen zu geringeren Anteilen eine freiwillige Tätigkeit ausüben.

\section{Abbildung 16-3: Anteile freiwillig Engagierter 2014, nach Erwerbsstatus}

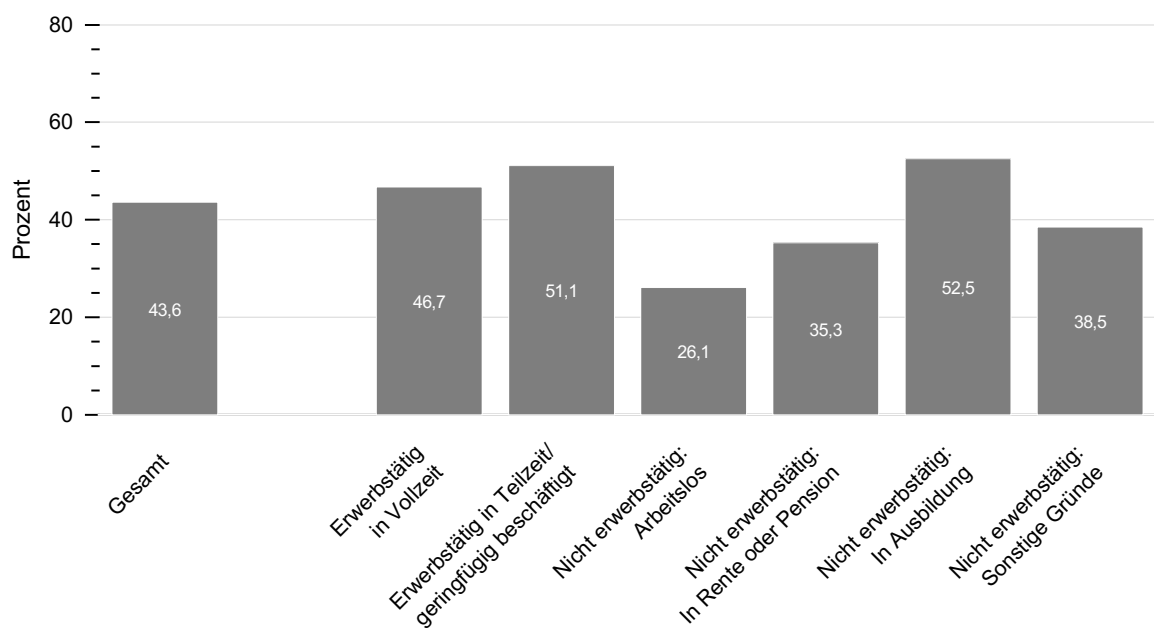

Quelle: FWS 2014, gewichtet, eigene Berechnungen (DZA). Basis: Alle Befragten ( $n=28.590)$. 
Abbildung 16-4: Anteile freiwillig Engagierter 2014, nach Erwerbsstatus und Geschlecht

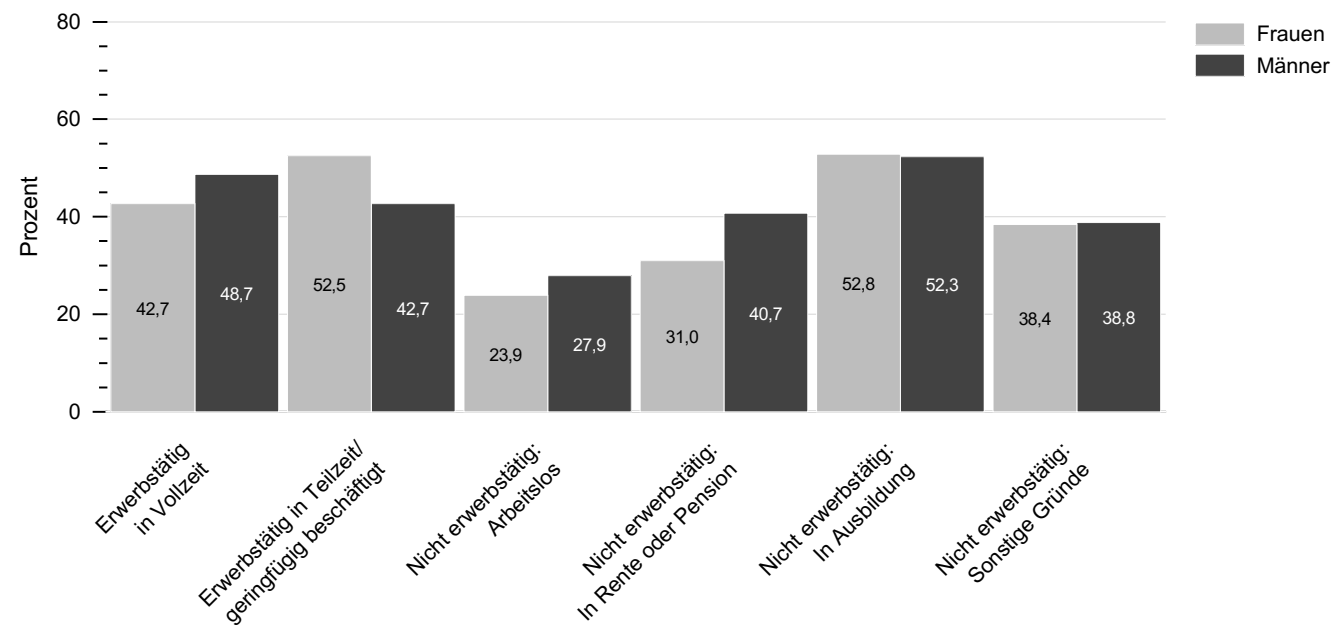

Quelle: FWS 2014, gewichtet, eigene Berechnungen (DZA). Basis: Alle Befragten ( $n=28.590)$. 


\subsection{Haushaltskonstellation und freiwilliges Engagement}

Neben der Erwerbstätigkeit können auch die familiäre Situation und die jeweilige Haushaltszusammensetzung freiwillige Tätigkeiten ermöglichen, aber auch erschweren. Im Folgenden soll daher betrachtet werden, wie das freiwillige Engagement mit der Haushaltskonstellation zusammenhängt.

In Kapitel 3 dieses Berichts wurde bereits dargestellt, wie sich Personen mit und ohne eigene Kinder im Haushalt sowie mit und ohne Partnerin oder Partner im Haushalt hinsichtlich ihrer Beteiligung im freiwilligen Engagement unterscheiden. Dieser Aspekt wird im Folgenden noch einmal aufgegriffen und differenziert nach unterschiedlichen Formen der Haushaltskonstellation betrachtet. Es zeigt sich, dass sich Personen, die alleine in Einpersonenhaushalten leben, mit 32,5 Prozent zu deutlich geringeren Anteilen engagieren als Personen in Mehrpersonenhaushalten (Abbildung 16-5). Die höchsten Anteile freiwillig Engagierter finden wir mit 54,9 Prozent bei Personen, die mit ihrer Partnerin beziehungsweise ihrem Partner und mindestens einem minderjährigen Kind zusammenleben. Menschen, die mit eigenen Kindern, aber ohne Partnerin beziehungsweise Partner oder aber ohne eigene Kinder leben, engagieren sich demgegenüber zu deutlich geringeren Anteilen.

\section{Abbildung 16-5: Anteile freiwillig Engagierter 2014, nach Haushaltskonstellation}

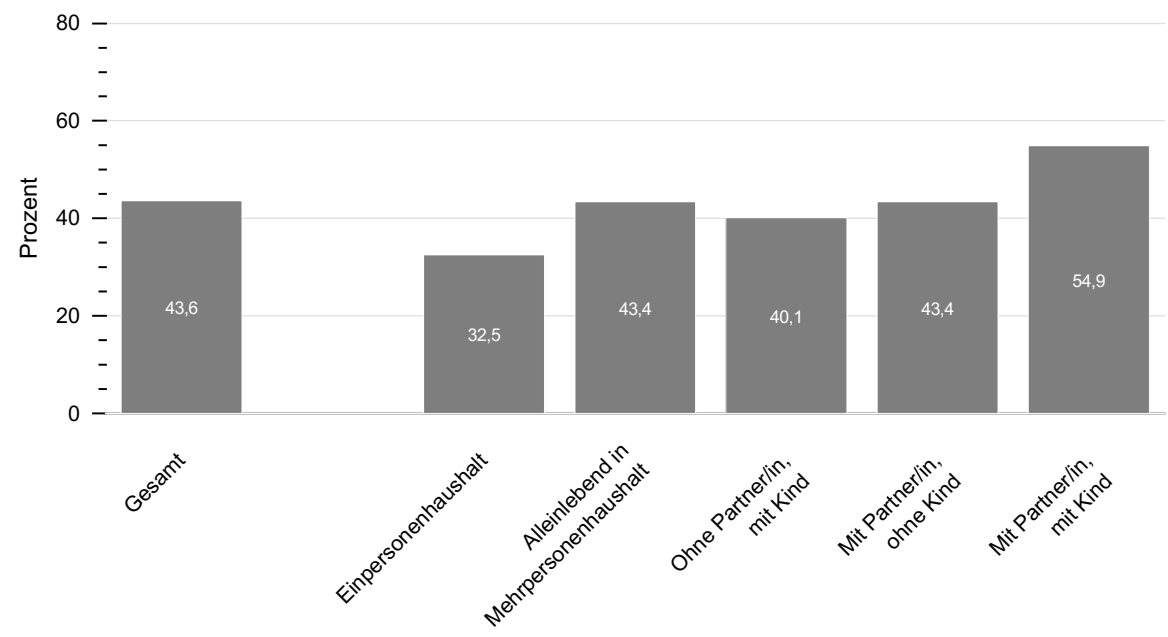

Quelle: FWS 2014, gewichtet, eigene Berechnungen (DZA). Basis: Alle Befragten $(n=27.276)$.

Bei der zusätzlichen Differenzierung nach Geschlecht (Abbildung 16-6) zeigt sich, dass Frauen in fast allen Haushaltskonstellationen zu geringeren Anteilen einer freiwilligen Tätigkeit nachgehen als Männer, die einzige Ausnahme bilden
Mehrpersonenhaushalte mit der Partnerin beziehungsweise dem Partner und einem minderjährigen Kind beziehungsweise Kindern - hier engagieren sich 56,7 Prozent der Frauen gegenüber 53,1 Prozent der Männer. 
Abbildung 16-6: Anteile freiwillig Engagierter 2014, nach Haushaltskonstellation und Geschlecht

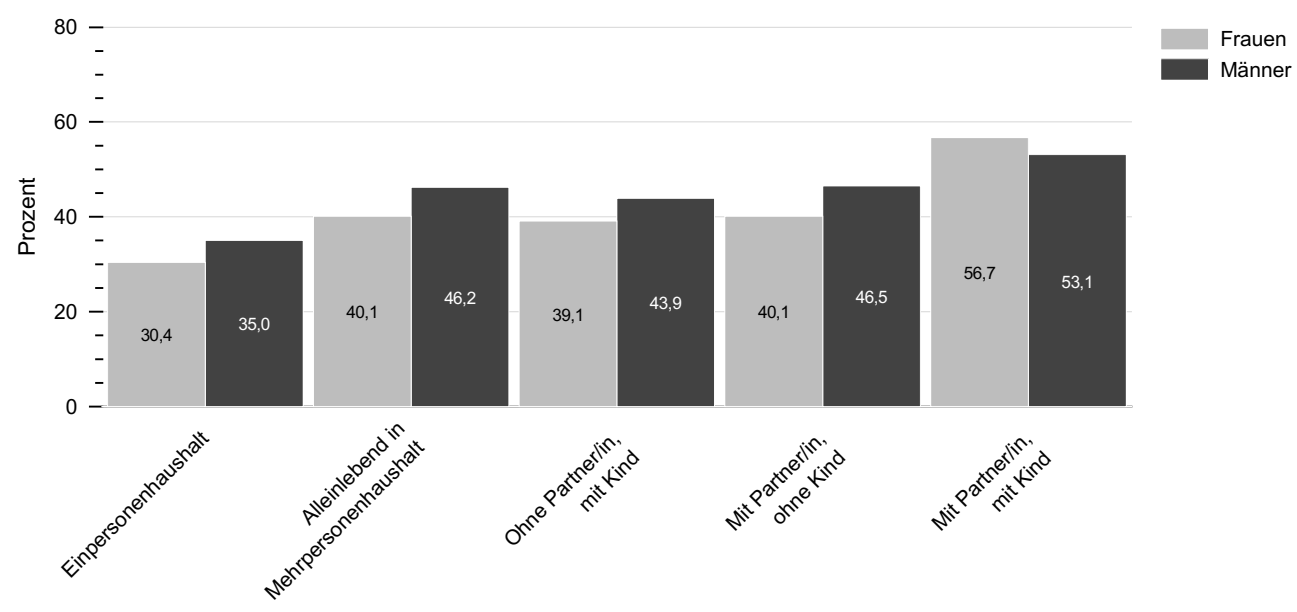

Quelle: FWS 2014, gewichtet, eigene Berechnungen (DZA). Basis: Alle Befragten $(n=27.276)$.

Abbildung 16-7: Anteile freiwillig Engagierter 2014, nach Haushaltskonstellation und Alter

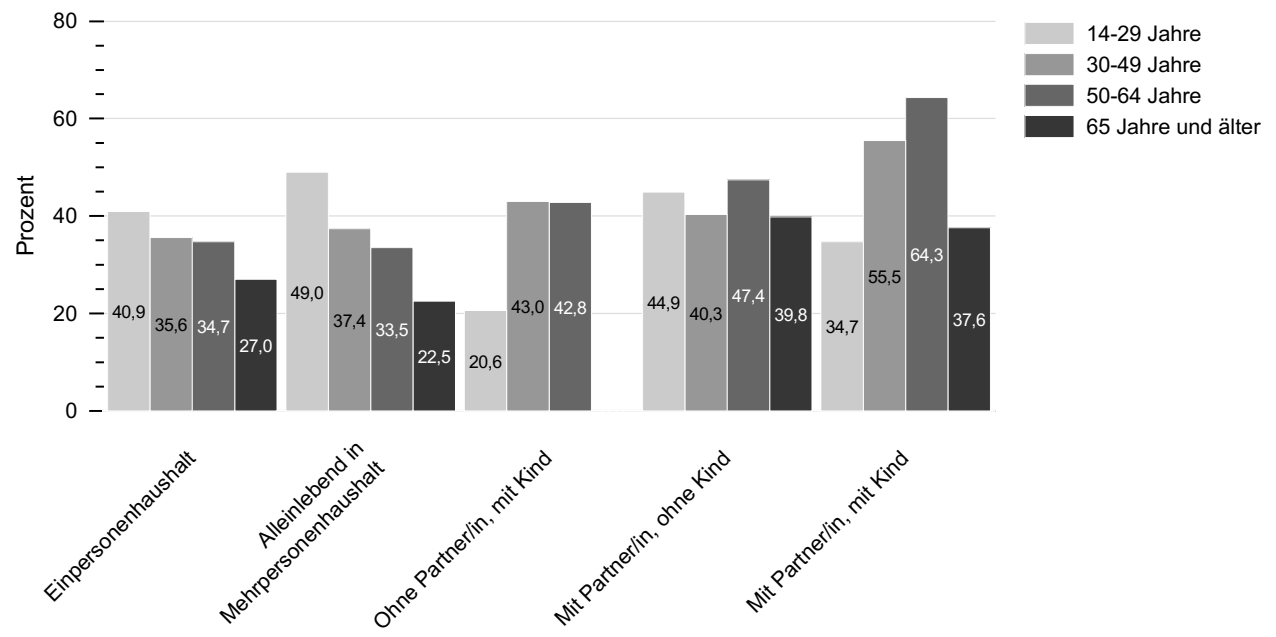

Quelle: FWS 2014, gewichtet, eigene Berechnungen (DZA). Basis: Alle Befragten $(n=27.276)$.

Die Kategorie ohne Partner/in mit Kind wird in der Altersgruppe 65 Jahre und älter wegen geringer Fallzahl in der Prozentuierungsbasis $(n<50)$ nicht ausgewiesen.

Die zusätzliche Betrachtung nach Altersgruppen (Abbildung 16-7) macht deutlich, dass sich in Einpersonenhaushalten und Mehrpersonenhaushalten ohne Partnerin beziehungsweise
Partner und ohne minderjährige Kinder 14- bis 29-Jährige zu höheren Anteilen engagieren als die übrigen Altersgruppen, während ihre Engagementquote in Haushalten mit Kindern (mit 
und ohne Partnerin beziehungsweise Partner) deutlich unterdurchschnittlich ist. Dies hängt vermutlich mit dem noch sehr geringen Alter der eigenen Kinder und den damit verbundenen geringen Zeitressourcen zusammen. Bei Personen im Alter von 30 bis 49 Jahren und 50 bis 64 Jahren, die mit ihren minderjährigen Kindern zu- sammenleben, sind die Kinder im Durchschnitt bereits deutlich älter, bedürfen also weniger Betreuung. Dies macht sich auch in den deutlich höheren Engagementraten von Personen dieser Altersgruppen, die mit ihren Kindern zusammenleben, bemerkbar.

\subsection{Einkommenssituation und freiwilliges Engagement}

Die materielle Situation ist relevant für die Ausübung freiwilliger und ehrenamtlicher Tätigkeiten. Abbildung 16-8 zeigt, dass freiwilliges Engagement deutlich häufiger ausgeübt wird, wenn Personen ihre finanziellen Ressourcen als gut bewerten. Nahezu jede zweite Person, die ihre eigene finanzielle Lage als sehr gut oder gut einschätzt, engagiert sich. Bei den Personen mit einer als sehr schlecht eingeschätzten finanziellen Lage engagiert sich dagegen nur etwa jede beziehungsweise jeder Vierte (26,9 Prozent).
Personen, die ihre finanzielle Situation als mittel oder eher schlecht einschätzen, liegen mit 39,6 Prozent beziehungsweise 37,9 Prozent dazwischen.

Dieser Zusammenhang zwischen finanzieller Situation und Engagement zeigt sich in ähnlicher Weise sowohl bei Frauen als auch bei Männern (Abbildung 16-9), wobei sich Frauen, die ihre finanzielle Lage als sehr schlecht einschätzen, zu besonders geringen Anteilen freiwillig engagieren (24,0 Prozent).

\section{Abbildung 16-8: Anteile freiwillig Engagierter 2014, nach finanzieller Situation}

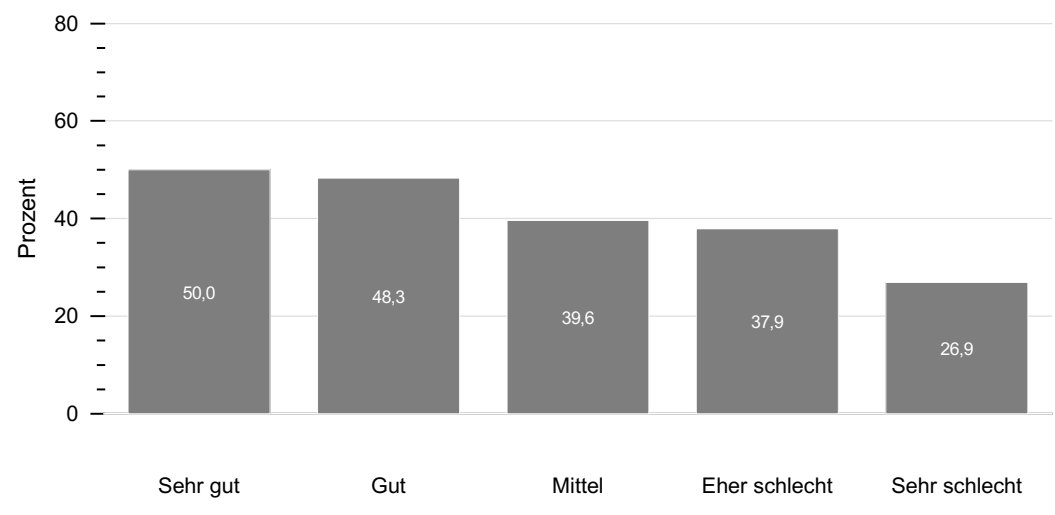

Quelle: FWS 2014, gewichtet, eigene Berechnungen (DZA). Basis: Alle Befragten ( $n=28.413$ ). 
Abbildung 16-9: Anteile freiwillig Engagierter 2014, nach finanzieller Situation und Geschlecht

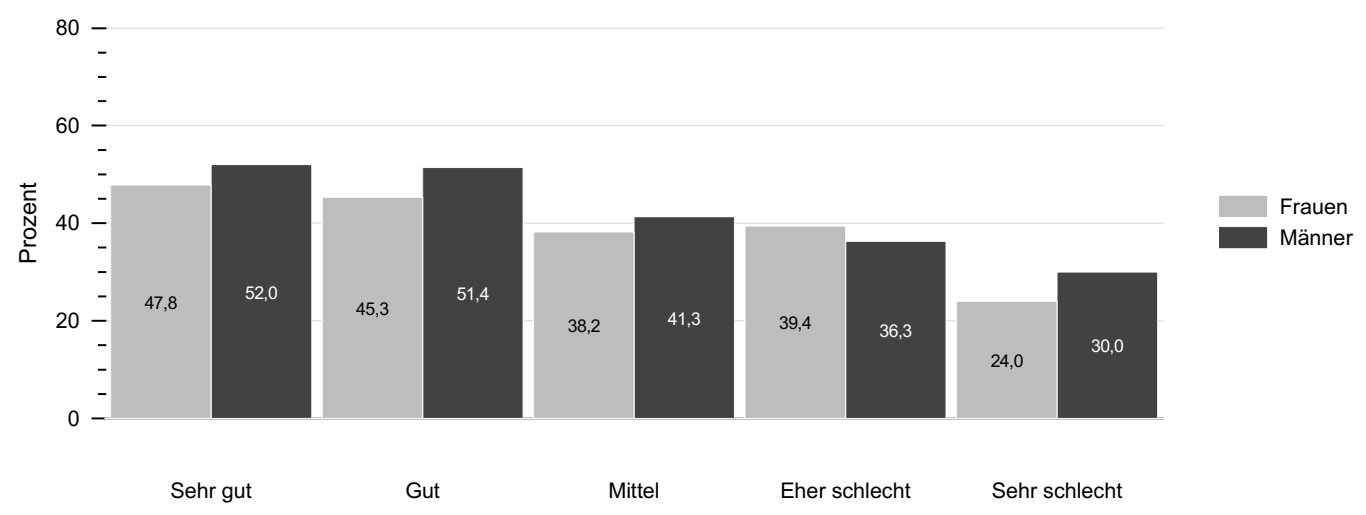

Quelle: FWS 2014, gewichtet, eigene Berechnungen (DZA). Basis: Alle Befragten $(n=28.413)$.

Darüber hinaus zeigt sich, dass sich in allen Altersgruppen Personen, die ihre finanzielle Lage als schlecht oder sehr schlecht einschätzen, zu geringeren Anteilen engagieren als Personen, die ihre finanzielle Situation besser bewerten (Abbildung 16-10). Deutlich wird aber auch, dass sich
Menschen ab 65 Jahren auch bei einer als gut eingeschätzten finanziellen Lage deutlich seltener engagieren als Jüngere. Ausgesprochen niedrige Engagementraten finden wir bei den Personen ab 65 Jahren, die ihre finanzielle Situation als sehr schlecht bewerten (17,7 Prozent).

\section{Abbildung 16-10: Anteile freiwillig Engagierter 2014, nach finanzieller Situation und Alter}

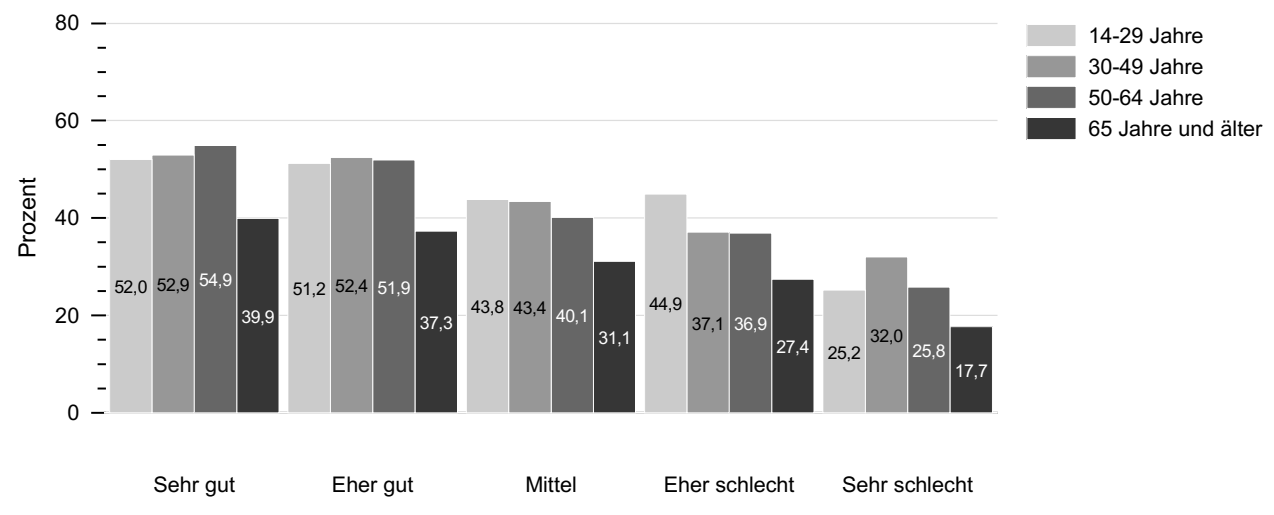

Quelle: FWS 2014, gewichtet, eigene Berechnungen (DZA). Basis: Alle Befragten $(n=28.413$ ). 


\subsection{Multivariate Analysen zu sozioökonomischen Faktoren und Engagement}

In den bisherigen Ergebnissen kam bereits zum Ausdruck, dass freiwilliges Engagement an Ressourcen geknüpft ist. Bei mangelnden Ressourcen, etwa wenn die finanzielle Situation als schlecht eingeschätzt wird, engagieren sich deutlich weniger Menschen als bei einer guten Ressourcenausstattung. Ähnliches trifft auf die schulische und berufliche Bildung zu. Auch der Erwerbsstatus und die Haushaltszusammensetzung hängen mit freiwilligem Engagement zusammen: Sie können Gelegenheiten für freiwillige Tätigkeiten schaffen, aber auch freiwilliges Engagement begrenzen, zum Beispiel wenn die Erwerbsarbeit in zeitlicher Konkurrenz zum Engagement steht. Sozioökonomische Faktoren wirken jedoch nicht isoliert, sondern sind im Zusammenspiel mit anderen Faktoren $\mathrm{zu}$ betrachten. Im Folgenden soll daher im Rahmen multivariater Modelle geprüft werden, wie die unterschiedlichen sozioökonomischen Aspekte unter Kontrolle weiterer individueller Merkmale auf freiwilliges Engagement wirken. Zudem ist $\mathrm{zu}$ fragen, ob sich diese Effekte auch finden lassen, wenn weitere Aspekte der Lebenssituation von Personen berücksichtigt werden, wie etwa die gesundheitliche Situation, die soziale Einbettung und Werthaltungen. Hierzu sind multivariate Analysen notwendig, in denen der Zusammenhang zwischen Engagement und mehreren Einflussfaktoren gleichzeitig untersucht wird.

Aus diesem Grund werden in diesem Abschnitt die Ergebnisse multivariater logistischer Regressionen dargestellt. In multivariaten logistischen Regressionsmodellen wird die Frage untersucht, ob eine abhängige Variable mit zwei Ausprägungen von mehreren unabhängigen Variablen beeinflusst wird. Die abhängige Variable in allen Modellen ist Engagement (ja/nein). Es wird also untersucht, wie sich das Vorliegen eines bestimmten Merkmals oder einer Merkmalskombination auf die Wahrscheinlichkeit auswirkt, sich freiwillig zu engagieren. Für jede der vier bislang betrachteten sozioökonomischen Variablen (schulische und berufliche Bildung, Erwerbsstatus, Haushaltskonstellation, subjektive Einkommenssituation) wird jeweils ein Modell gerechnet. In diese Modelle gehen als unabhängige Variablen jeweils eine der vier sozioökonomischen Variablen ein, zudem Geschlecht und Alter sowie die Interaktionen zwischen Geschlecht mit der jeweiligen Variablen. Als Referenzgruppe für die Testung der Effekte wurde die Gruppe der Männer im Alter von 50 bis 64 Jahren gewählt. Die Gruppe der Männer wurde als Referenzgröße gewählt, um die Ergebnisse für die Gruppe der Frauen explizit darstellen zu können (wird die Referenzgruppe Frauen gewählt, kann kein Koeffizient für Frauen ausgewiesen werden). Das Alter 50 bis 64 Jahre wurde gewählt, da es in einer mittleren Position liegt. Als Kontrollvariablen gehen in die Analysen darüber hinaus die folgenden Informationen ein: subjektive Gesundheit, Netzwerkqualität, Werthaltung Solidarität sowie Bundesland und Kontakt über Festnetz/Mobilfunk. Zusätzlich wurde ein Modell gerechnet, das alle vier sozioökonomischen Variablen dieses Kapitels sowie Geschlecht und Alter und die genannten Kontrollvariablen enthält, jedoch keine Interaktionen.

In den Ergebnistabellen sind die unstandardisierten Koeffizienten der jeweiligen Modelle dargestellt. Positive Koeffizienten (z. B. im Fall der Variable ,Bildungsniveau nach ISCED‘ mit der Ausprägung, noch Schüler/Schülerin': 0,42) zeigen an, dass eine positive Beziehung vorliegt: Bei Schülerinnen und Schülern ist die Wahrscheinlichkeit, freiwillig engagiert zu sein, höher als in der Referenzgruppe derjenigen, die einen Haupt- oder Realschulabschluss und eine berufliche Ausbildung haben. Negative Koeffizienten (z. B. im Fall der Variable ,Bildungsniveau nach ISCED‘ mit der Ausprägung ,Haupt-/Realschulabschluss ohne berufliche Ausbildung: -0,32) zeigen an, dass eine negative Beziehung vorliegt: 
Bei Personen, die einen Haupt- oder Realschulabschluss ohne Berufsausbildung haben, ist die Wahrscheinlichkeit, sich freiwillig zu engagieren, geringer als in der Referenzgruppe derjenigen, die einen Haupt- oder Realschulabschluss und eine berufliche Ausbildung haben. Je größer der
Betrag eines Koeffizienten ist, desto stärker ist der Zusammenhang. Dargestellt sind nur jene Koeffizienten, bei denen ein Signifikanztest angezeigt hat, dass der betreffende Koeffizient statistisch bedeutsam ist (nicht signifikante Koeffizienten werden durch drei Striche angezeigt).

Tabelle 16-7: Ergebnisse logistischer Regression, Vorhersage freiwilligen Engagements durch Bildungsniveau nach ISCED-Klassifizierung, Geschlecht, Alter sowie Interaktionen, 2014

\begin{tabular}{|c|c|}
\hline \multicolumn{2}{|l|}{$\begin{array}{l}\text { Bildung nach ISCED (Referenz: Haupt-/Realschulabschluss } \\
\text { und berufliche Ausbildung) }\end{array}$} \\
\hline Noch Schüler/Schülerin & $0,42 * * *$ \\
\hline Haupt-/Realschulabschluss ohne berufliche Ausbildung & $-0,32 * * *$ \\
\hline FH-Reife/Abitur ohne berufliche Ausbildung & $0,31 * * *$ \\
\hline FH-Reife/Abitur und berufliche Ausbildung & $0,19 *$ \\
\hline Abschluss Berufs-/Fachakademie & $0,52 * * *$ \\
\hline FH-/Universitätsabschluss & $0,62 * * *$ \\
\hline \multicolumn{2}{|l|}{ Geschlecht (Referenz: Männer) } \\
\hline Frauen & $-0,27 * * *$ \\
\hline \multicolumn{2}{|l|}{ Alter (Referenz: 50 bis 64 Jahre) } \\
\hline 14 bis 29 Jahre & --- \\
\hline 30 bis 49 Jahre & $0,11^{* *}$ \\
\hline 65 Jahre und älter & $-0,30 * * *$ \\
\hline \multicolumn{2}{|l|}{ Interaktion mit Geschlecht } \\
\hline Frau*Noch Schüler/Schülerin & --- \\
\hline Frau*Haupt-/Realschulabschluss ohne berufliche Ausbildung & --- \\
\hline Frau*FH-Reife/Abitur ohne berufliche Ausbildung & --- \\
\hline Frau*FH-Reife/Abitur und berufliche Ausbildung & $0,22 *$ \\
\hline \multicolumn{2}{|l|}{ Frau*Abschluss Berufs-/Fachakademie } \\
\hline Frau*FH-/Universitätsabschluss & --- \\
\hline Pseudo $\mathrm{R}^{2}$ & $0,06 * * *$ \\
\hline Anzahl gültiger Fälle & 28.071 \\
\hline
\end{tabular}

Quelle: FWS 2014, ungewichtet, eigene Berechnungen (DZA). Kontrolliert für Netzwerkqualität, Werthaltung Solidarität, subjektive Gesundheit sowie Design-Variablen (Bundesland, Kontakt über Festnetz/Mobilfunk). ${ }^{*} p<0,05,{ }^{*} p<0,01, * * * p<0,001,---$ nicht signifikant.

Tabelle 16-7 enthält das Ergebnis einer logistischen Regression zur Vorhersage freiwilligen Engagements durch das Bildungsniveau nach
ISCED-Klassifizierung. Das Bildungsniveau hat auch bei Kontrolle weiterer Merkmale einen Einfluss auf die Wahrscheinlichkeit sich freiwillig 
$\mathrm{zu}$ engagieren. Bei allen Bildungsgruppen, mit Ausnahme derjenigen, die über einen Hauptoder Realschulabschluss ohne Berufsausbildung verfügen, ist die Wahrscheinlichkeit sich $\mathrm{zu}$ engagieren, höher als bei denjenigen, die einen Haupt- oder Realschulabschluss sowie eine Berufsausbildung haben. Die Koeffizienten für die Altersgruppen entsprechen den bereits aus den deskriptiven Analysen bekannten Befunden. Frauen sind, auch bei Einbezug der Schul- und
Berufsbildung, mit geringerer Wahrscheinlichkeit engagiert als Männer. Der positive Interaktionseffekt zwischen dem Geschlecht und dem Vorliegen von Abitur beziehungsweise Fachhochschulreife und einer beruflichen Ausbildung zeigt an, dass dieses Bildungsniveau die Wahrscheinlichkeit sich zu engagieren bei Frauen deutlicher erhöht als bei Männern. Für die anderen Bildungsstufen zeigen sich keine Interaktionen mit dem Geschlecht.

Tabelle 16-8: Ergebnisse logistischer Regression, Vorhersage freiwilligen Engagements durch Erwerbsstatus, Geschlecht, Alter sowie Interaktionen, 2014

\begin{tabular}{lc}
\hline Erwerbsstatus (Referenz: Erwerbstätig in Vollzeit) & --- \\
\hline Erwerbstätig in Teilzeit/geringfügig & $-0,39^{* * *}$ \\
\hline Arbeitslos & --- \\
\hline Rentner/-in & $0,88^{* * *}$ \\
\hline In Aus-/Weiterbildung & --- \\
\hline Nicht erwerbstätig, sonstige Gründe & \\
\hline Geschlecht (Referenz: Männer) & $-0,36^{* * *}$ \\
\hline Frauen & --- \\
\hline Alter (Referenz: 50 bis 64 Jahre) & $0,11^{* *}$ \\
\hline 14 bis 29 Jahre & $-0,31^{* * *}$ \\
\hline 30 bis 49 Jahre & \\
\hline 65 Jahre und älter & $0,38^{* *}$ \\
\hline Interaktion mit Geschlecht & --- \\
\hline Frau*Erwerbstätig in Teilzeit/geringfügig & --- \\
\hline Frau*Arbeitslos & $0,25^{* *}$ \\
\hline Frau*Rentner/-in & $--06^{* * *}$ \\
\hline Frau*In Aus-/Weiterbildung & 28.035 \\
\hline Frau*Nicht erwerbstätig, sonstige Gründe & \\
\hline Pseudo R2 & \\
\hline Anzahl gültiger Fälle & \\
\hline
\end{tabular}

Quelle: FWS 2014, ungewichtet, eigene Berechnungen (DZA). Kontrolliert für Schul- und Berufsbildung nach ISCED, Netzwerkqualität, Werthaltung Solidarität, subjektive Gesundheit sowie Design-Variablen (Bundesland, Kontakt über Festnetz/Mobilfunk). ${ }^{*} p<0,05,{ }^{* *} p<0,01,{ }^{* * *} p<0,001,---$ nicht signifikant.

Auch der Erwerbsstatus wirkt sich im multivariaten Modell auf die Wahrscheinlichkeit eines freiwilligen Engagements aus (Tabelle 16-8).
Arbeitslose sind mit geringerer und Personen in Aus- oder Weiterbildung mit höherer Wahrscheinlichkeit freiwillig engagiert als Erwerbs- 
tätige in Vollzeit. In Teilzeit oder geringfügig erwerbstätig zu sein, erhöht nur bei Frauen die Wahrscheinlichkeit für ein freiwilliges Engagement. Außerdem ist der Effekt des Erwerbsstatus ,Aus- oder Weiterbildung' bei Frauen ausgeprägter.

Deutliche Effekte zeigen sich auch für die Haushaltskonstellation (Tabelle 16-9). Wie bereits in der deskriptiven Betrachtung festgestellt, ist die Wahrscheinlichkeit einer freiwilligen Tätigkeit für alle Haushaltstypen höher als für Einpersonenhaushalte. Allerdings gibt es auch hier wieder geschlechtsspezifische Effekte: Unter allen Personen, die ohne Partner/-in und minderjährigem Kind, aber mit anderen Personen zusammenwohnen, ist es für Frauen weniger wahrscheinlich einem Engagement nachzugehen als für Männer. Dagegen macht es bei Frauen für die Frage, ob man sich engagiert oder nicht, einen größeren Unterschied, wenn man mit Partnerin beziehungsweise Partner und Kind(ern) zusammenlebt, als bei Männern. Die Haupteffekte für Geschlecht und Alter entsprechen auch hier den deskriptiven Befunden.

Tabelle 16-9: Ergebnisse logistischer Regression, Vorhersage freiwilligen Engagements durch Haushaltskonstellation, Geschlecht, Alter sowie Interaktionen, 2014

\begin{tabular}{|c|c|}
\hline \multicolumn{2}{|c|}{ Haushaltskonstellation (Referenz: Einpersonenhaushalt) } \\
\hline Alleinlebend in Mehrpersonenhaushalt & $0,41 * * *$ \\
\hline Ohne Partner/in, mit Kind & $0,64^{* *}$ \\
\hline Mit Partner/in, ohne Kind & $0,27^{* * *}$ \\
\hline Mit Partner/in, mit Kind & $0,60 * * *$ \\
\hline \multicolumn{2}{|l|}{ Geschlecht (Referenz: Männer) } \\
\hline Frauen & $-0,18 * * *$ \\
\hline \multicolumn{2}{|l|}{ Alter (Referenz: 50 bis 64 Jahre) } \\
\hline 14 bis 29 Jahre & $0,17^{* *}$ \\
\hline 30 bis 49 Jahre & $-0,10^{*}$ \\
\hline 65 Jahre und älter & $-0,25 * * *$ \\
\hline \multicolumn{2}{|l|}{ Interaktion mit Geschlecht } \\
\hline Frau*Alleinlebend in Mehrpersonenhaushalt & $-0,20 *$ \\
\hline Frau*Ohne Partner/in, mit Kind & --- \\
\hline Frau*Mit Partner/in, ohne Kind & --- \\
\hline Frau*Mit Partner/in, mit Kind & $0,21^{*}$ \\
\hline Pseudo $\mathrm{R}^{2}$ & 0,07 \\
\hline Anzahl gültiger Fälle & 26.682 \\
\hline
\end{tabular}

Quelle: FWS 2014, ungewichtet, eigene Berechnungen (DZA). Kontrolliert für Schul- und Berufsbildung nach ISCED, Netzwerkqualität, Werthaltung Solidarität, subjektive Gesundheit sowie Design-Variablen (Bundesland, Kontakt über Festnetz/Mobilfunk). ${ }^{*} p<0,05,{ }^{*} p<0,01, * * * p<0,001,---$ nicht signifikant. 
Tabelle 16-10 stellt die Ergebnisse eines Modells dar, in dem freiwilliges Engagement durch die subjektive Einkommenssituation vorhergesagt wird. Personen, die ihre finanzielle Situation als gut oder sehr gut bewerten, engagieren sich mit höherer Wahrscheinlichkeit als Personen, die ihre Situation schlechter einschätzen. Die Haupteffekte für Geschlecht und Alter weisen in die erwarteten Richtungen. Eine Interaktion zwischen der finanziellen Situation und dem Geschlecht zeigt sich nicht.

Tabelle 16-10: Ergebnisse logistischer Regression, Vorhersage freiwilligen Engagements durch subjektive Einkommenssituation, Geschlecht, Alter sowie Interaktionen, 2014

\begin{tabular}{|c|c|}
\hline \multicolumn{2}{|c|}{ Subjektive Einkommenssituation (Referenz: Mittel und schlechter) } \\
\hline Gut/sehr gut & $0,19 * * *$ \\
\hline \multicolumn{2}{|c|}{ Geschlecht (Referenz: Männer) } \\
\hline Frauen & $-0,20 * * *$ \\
\hline \multicolumn{2}{|c|}{ Alter (Referenz: 50 bis 64 Jahre) } \\
\hline 14 bis 29 Jahre & $0,39 * * *$ \\
\hline 30 bis 49 Jahre & $0,13 * * *$ \\
\hline 65 Jahre und älter & $-0,31 * * *$ \\
\hline \multicolumn{2}{|l|}{ Interaktion mit Geschlecht } \\
\hline Frauen*Gut/sehr gut & --- \\
\hline Pseudo $\mathrm{R}^{2}$ & 0,05 \\
\hline Anzahl gültiger Fälle & 27.862 \\
\hline
\end{tabular}

Quelle: FWS 2014, ungewichtet, eigene Berechnungen (DZA). Kontrolliert für Schul- und Berufsbildung nach ISCED, Netzwerkqualität, Werthaltung Solidarität, subjektive Gesundheit sowie Design-Variablen (Bundesland, Kontakt über Festnetz/Mobilfunk). ${ }^{*} p<0,05,{ }^{*} p<0,01, * * * p<0,001,---$ nicht signifikant.

Bleiben die Effekte der sozioökonomischen Faktoren bestehen, wenn man für die jeweils anderen Aspekte kontrolliert? Das in Tabelle 16-11 dargestellte Modell zeigt, dass dies der Fall ist. Die Effekte für die schulische und berufliche Bildung, den Erwerbsstatus und die Haushaltskonstellation bleiben auch bei Einbezug der jeweils anderen Variablen weitgehend stabil. Lediglich der Zusammenhang zwischen der finanziellen Situation und Engagement ist bei gleichzeitiger Betrachtung aller sozioökonomischen Variablen nicht mehr statistisch signifikant. Die berück- sichtigten sozioökonomischen Faktoren wirken also größtenteils auch kumulativ. Die Wahrscheinlichkeit sich freiwillig zu engagieren sinkt, wenn ungünstige Ressourcenausstattungen (beispielsweise ein niedriger Bildungsstatus und Arbeitslosigkeit) zusammentreffen und steigt, wenn förderliche Ressourcen beziehungsweise Gelegenheitsstrukturen für Engagement aufeinandertreffen (beispielsweise eine hohe schulische und berufliche Bildung und ein Zusammenleben mit Kindern). 
Tabelle 16-11: Ergebnisse logistischer Regression, Vorhersage freiwilligen Engagements durch Schul- und Berufsbildung nach ISCED, Erwerbsstatus, Haushaltskonstellation, Subjektive Einkommenssituation, Geschlecht, Alter, 2014

\begin{tabular}{|c|c|}
\hline \multicolumn{2}{|c|}{ Bildung nach ISCED (Referenz: Haupt-/Realschulabschluss und berufliche Ausbildung) } \\
\hline Noch Schüler/Schülerin & --- \\
\hline Haupt-/Realschulabschluss ohne berufliche Ausbildung & $-0,55 * * *$ \\
\hline FH-Reife/Abitur ohne berufliche Ausbildung & --- \\
\hline FH-Reife/Abitur und berufliche Ausbildung & $0,32 * * *$ \\
\hline Abschluss Berufs-/Fachakademie & $0,52 * * *$ \\
\hline FH-/Universitätsabschluss & $0,67 * * *$ \\
\hline \multicolumn{2}{|l|}{ Erwerbsstatus (Referenz: Erwerbstätig in Vollzeit) } \\
\hline Erwerbstätig in Teilzeit/geringfügig & $0,21 * * *$ \\
\hline Arbeitslos & $-0,29 * *$ \\
\hline Rentner/-in & --- \\
\hline In Aus-/Weiterbildung & $0,73 * * *$ \\
\hline Nicht erwerbstätig, sonstige Gründe & --- \\
\hline \multicolumn{2}{|l|}{ Haushaltskonstellation (Referenz: Einpersonenhaushalt) } \\
\hline Alleinlebend in Mehrpersonenhaushalt & $0,14^{* *}$ \\
\hline Ohne Partner/in, mit Kind & $0,39 * * *$ \\
\hline Mit Partner/in, ohne Kind & $0,19 * * *$ \\
\hline Mit Partner/in, mit Kind & $0,70 * * *$ \\
\hline \multicolumn{2}{|c|}{ Subjektive Einkommenssituation (Referenz: Mittel und schlechter) } \\
\hline Gut/Sehr gut & --- \\
\hline \multicolumn{2}{|l|}{ Geschlecht (Referenz: Männer) } \\
\hline Frauen & $-0,24 * * *$ \\
\hline \multicolumn{2}{|l|}{ Alter (Referenz: 50 bis 64 Jahre) } \\
\hline 14 bis 29 Jahre & --- \\
\hline 30 bis 49 Jahre & $-0,10^{*}$ \\
\hline 65 Jahre und älter & $-0,28 * * *$ \\
\hline Pseudo $\mathrm{R}^{2}$ & 0,07 \\
\hline Anzahl gültiger Fälle & 26.422 \\
\hline
\end{tabular}

Quelle: FWS 2014, ungewichtet, eigene Berechnungen (DZA). Kontrolliert für Netzwerkqualität, Werthaltung Solidarität, subjektive Gesundheit sowie Design-Variablen (Bundesland, Kontakt über Festnetz/Mobilfunk). ${ }^{*} p<0,05,{ }^{* *} p<0,01$, $* * * p<0,001,---$ nicht signifikant. 
Abschließend ist darauf hinzuweisen, dass die Erklärungskraft der multivariaten Modelle nicht besonders hoch ist, wie an der aufgeklärten Varianz (Pseudo $\mathrm{R}^{2}$ ) abgelesen werden kann: Sie liegt bei den in diesem Kapitel präsentierten Modellen zwischen fünf und sieben Prozent.
Dies ist auch auf die Vielfältigkeit des freiwilligen Engagements zurückzuführen, welches sehr unterschiedliche Aktivitäten und Aufgaben umfassen kann, die von den Einflussgrößen in unterschiedlicher Weise beeinflusst werden.

\subsection{Fazit}

Personen mit geringeren sozioökonomischen Ressourcen engagieren sich zu geringeren Anteilen als Personen mit besserer Ressourcenausstattung. Dies zeigt sich hinsichtlich der schulischen und beruflichen Bildung, des Erwerbsstatus sowie der subjektiven Einkommenssituation. Darüber hinaus kann auch die Haushaltskonstellation Gelegenheiten, aber auch Hindernisse für freiwilliges Engagement beinhalten. Die sozioökonomischen Faktoren wirken auch, wenn weitere Merkmale der Lebenssituation einbezogen werden, und sie wirken kumulativ. Das heißt, die Wahrscheinlichkeit sich freiwillig $\mathrm{zu}$ engagieren sinkt, wenn ungünstige Ressourcenausstattungen wie beispielsweise ein niedriger Bildungsstatus und Arbeitslosigkeit zusammenkommen, und sie steigt, wenn förderliche Ressourcen aufeinandertreffen.

Hinter den geringeren Engagementquoten bei Personen mit niedrigerem sozioökonomischen Status können sich ungleiche Zugangschancen verbergen. Daher sollte überlegt werden, mit welchen Angeboten Personen mit niedrigen sozioökonomischen Ressourcen erreicht werden können, um bessere Zugangschancen zu schaffen. Zu bedenken ist dabei allerdings, dass es auch Personengruppen gibt, die überhaupt nicht durch engagementfördernde Maßnahmen erreicht werden können, weil ihnen die Voraussetzungen fehlen, eine Aufgabe oder ein Amt zu übernehmen, oder weil sie kein Interesse daran haben. Schließlich ist eine gewisse materielle Absicherung eine Grundvoraussetzung um am gesellschaftlichen Leben teilnehmen zu können. Auch armutsvermeidende Maßnahmen können somit zu einer Verbesserung der Teilhabechancen durch freiwilliges Engagement beitragen.

Neben den finanziellen Möglichkeiten sind auch zeitliche Ressourcen im Zusammenspiel von Erwerbstätigkeit, Familie und Engagement $\mathrm{zu}$ berücksichtigen. Dies scheint insbesondere vor dem Hintergrund der unterschiedlichen Eingebundenheit von Frauen und Männern in familiale Aufgaben wichtig. Frauen investieren aufgrund der immer noch weit verbreiteten traditionellen geschlechtsspezifischen Arbeitsteilung häufig mehr Zeit in Haushalt und Kinderbetreuung als Männer. Die Zeitressourcen für freiwilliges Engagement sind bei erwerbstätigen Frauen also möglicherweise knapper als bei erwerbstätigen Männern. Hinzu kommen unterschiedliche Gelegenheitsstrukturen für freiwilliges Engagement durch Unterschiede in der Erwerbspartizipation. Die Gleichstellung von Frauen und Männern im Engagement kann daher nur gelingen, wenn gleichzeitig auch die Gleichstellung der Geschlechter in Beruf und Familie angestrebt wird.

Im vorliegenden Kapitel wurde aufgezeigt, inwieweit sozioökonomische Faktoren förderlich oder hinderlich für freiwilliges Engagement sind. Auf der anderen Seite kann sich aber auch freiwilliges Engagement förderlich auf den sozioökonomischen Status einer Person auswirken, beispielsweise wenn bei einer freiwilligen Tätigkeit Qualifikationen erworben werden, die auch im Beruf eingesetzt werden können (siehe Kapitel 13), oder aber wenn im Engagement Kontakte geknüpft werden, die die Beschäftigungschancen auf dem Arbeitsmarkt erhöhen. Gerade vor dem 
Hintergrund dieser sich aus dem Engagement ergebenden Möglichkeiten hinsichtlich der Stellung im sozialen Gefüge einer Gesellschaft, aber auch unter dem Gesichtspunkt der Ermöglichung guter gesellschaftlicher Teilhabechancen aller Bevölkerungsschichten scheint ein Abbau von Barrieren für freiwilliges Engagement für Personen mit geringen sozioökonomischen Ressourcen ratsam.

\section{Literatur}

Alscher, M. (2010). Auf der Suche nach Balance. Frauen und Männer zwischen Beruf, Familie und Engagement. WZB Mitteilungen, 2010(129), 31-33.

Böhnke, P. (2008). Are the poor socially integrated? The link between poverty and social support in different welfare regimes. Journal of European Social Policy, 18(2), 133-150.

Erlinghagen, M. (2008). Ehrenamtliche Arbeit und informelle Hilfe nach dem Renteneintritt. In: M. Erlinghagen \& K. Hank (Hrsg.) Produktives Altern und informelle Arbeit in modernen Gesellschaften (S. 93-118). Wiesbaden: VS Verlag für Sozialwissenschaften.

Gensicke, T. (2015). Freiwilliges Engagement in Deutschland: Freiwilligensurvey 2009. Wiesbaden: Springer VS.

Naumann, D., \& Romeu Gordo, L. (2010). Gesellschaftliche Partizipation: Erwerbstätigkeit, Ehrenamt und Bildung. In: A. Motel-Klingebiel, S. Wurm \& C. Tesch-Römer (Hrsg.) Altern im Wandel. Befunde des Deutschen Alterssurveys (DEAS) (S. 118-141). Stuttgart: Kohlhammer.

Organisation for Economic Co-operation and Development (OECD) (Hrsg.) (1999). Classifying Educational Programmes: Manual for ISCED-97 Implementation in OECD Countries. Paris: OECD.

Schmidt, B. O. (2014). Ehrenamtliches Engagement in Vereinen und Initiativen. In: U. Engel (Hrsg.) Gerechtigkeit ist gut, wenn sie mir nützt. Was den Deutschen wichtig ist (S. 131-148). Frankfurt am Main: Campus.

Simonson, J., Hagen, C., Vogel, C., \& Motel-Klingebiel, A. (2013). Ungleichheit sozialer Teilhabe im Alter. Zeitschrift für Gerontologie und Geriatrie, 46(5), 410-416.

Strauß, S. (2009). Ehrenamt in Deutschland und Großbritannien - Sprungbrett zurück auf den Arbeitsmarkt? Kölner Zeitschrift für Soziologie und Sozialpsychologie, 61(4), 647-670.

United Nations Statistics Division (UNSD) (2011). A New ISCED. Classifications Newsletter, August 2011. Online: http://www.uis.unesco.org/Education/Documents/UNSD_newsletter_27e_ISCED.pdf (zuletzt abgerufen 5.2.2016).

Zierau, J. (2001). Genderperspektive - Freiwilligenarbeit, ehrenamtliche Tätigkeit und bürgerschaftliches Engagement bei Männern und Frauen In: S. Picot (Hrsg.) Freiwilliges Engagement in Deutschland. Freiwilligensurvey 1999. Ergebnisse der Repräsentativerhebung zu Ehrenamt, Freiwilligenarbeit und bürgerschaftlichem Engagement. Band 3: Frauen und Männer, Jugend, Senioren, Sport (S. 15-110). Stuttgart: Kohlhammer.

Open Access Dieses Kapitel wird unter der Creative Commons Namensnennung 4.0 International Lizenz (http://creativecommons.org/licenses/by/4.0/deed.de) veröffentlicht, welche die Nutzung, Vervielfältigung, Bearbeitung, Verbreitung und Wiedergabe in jeglichem Medium und Format erlaubt, sofern Sie den/die ursprünglichen Autor(en) und die Quelle ordnungsgemäß nennen, einen Link zur Creative Commons Lizenz beifügen und angeben, ob Änderungen vorgenommen wurden.

Die in diesem Kapitel enthaltenen Bilder und sonstiges Drittmaterial unterliegen ebenfalls der genannten Creative Commons Lizenz, sofern sich aus der Abbildungslegende nichts anderes ergibt. Sofern das betreffende Material nicht unter der genannten Creative Commons Lizenz steht und die betreffende Handlung nicht nach gesetzlichen Vorschriften erlaubt ist, ist für die oben aufgeführten Weiterverwendungen des Materials die Einwilligung des jeweiligen Rechteinhabers einzuholen. 